\title{
Digital stethoscope: technology update
}

\section{Supreeya Swarup' Amgad N Makaryus ${ }^{1,2}$ \\ 'Department of Cardiology, Nassau University Medical Center, East Meadow, NY, ${ }^{2}$ Department of Cardiology, Zucker School of Medicine at Hofstra/Northwell Health, Hempstead, NY, USA}

Video abstract

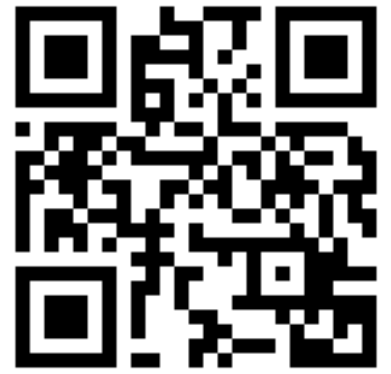

Point your SmartPhone at the code above. If you have a QR code reader the video abstract will appear. Or use: http://youtu.be/scDpoKBfkbw
Correspondence: Amgad N Makaryus Department of Cardiology, Nassau University Medical Center, 220I Hempstead Turnpike, East Meadow, NY II554, USA

Tel +I 5162964949

Fax +I 5165723172

Email amakaryu@numc.edu
This article was published in the following Dove Press journal:

Medical Devices: Evidence and Research

\begin{abstract}
Cardiovascular disease (CVD) is recognized as the leading cause of mortality throughout the world. About one-third of global mortality is attributable to CVD. In addition to clinical presentation, specific clinical exam findings can assist in treating and preventing CVD. CVD may initially manifest as pulmonary pathology, and thus, accurate cardiopulmonary auscultation is paramount to establishing accurate diagnosis. One of the most powerful tools available to physicians is the stethoscope. The stethoscope first emerged in the year 1818, invented by a French physician, René Laennec. Since then, the initial modest monaural wooden tube has evolved into a sophisticated digital device. This paper provides an analysis of the evolution of the stethoscope as well as highlights the advancement made by the modern digital stethoscope including the application of this tool in advancing care for patients suffering from CVD.
\end{abstract}

Keywords: cardiovascular disease, physical exam, diagnosis, treatment

\section{Introduction}

Cardiovascular disease (CVD) is recognized as the leading cause of mortality in countries throughout the world, and thus, timely detection, treatment, and prevention are the cornerstone of the comprehensive care that a physician provides to his/her patients. ${ }^{1}$ In $2015,31 \%$ of global mortality was attributable to CVD, where 17.7 million people died from a cardiac cause. ${ }^{1}$ Auscultation of the cardiovascular system can assist in timely diagnosis of valvular heart disease (VHD), congestive heart failure, hypertensive disease, arrhythmias such as atrial fibrillation, obstructive arterial disease, and structural heart disease among several others. Furthermore, multiple pulmonary diseases can manifest as right-sided heart disease and multiple heart diseases can first present as pulmonary pathology; thus, pulmonary auscultation and interpretation can further assist in forming accurate diagnosis.

Cardiovascular examination usually comprises the following: evaluation of the carotid arterial pulse and jugular venous pulse; auscultation for bruits; auscultation of venous hums, precordial impulses, and palpation of heart sounds and murmurs; auscultation of the heart sounds; and evaluation of the peripheral arterial and venous system. ${ }^{2}$ Auscultation of the heart sounds is the foundation upon which a physician can diagnose CVD and provide cost-effective means for additional tests. Auscultation represents recognition of mechanical vibration from the body surface at the frequency range of sound $(20-20,000 \mathrm{~Hz}){ }^{3}$ Vibrations below this frequency range are defined as "infrasonic" and are usually appreciated as thrusts, heaves, and arterial or venous pulsations. 
In the modern world, several modalities are used in addition to the clinical examination in evaluation of CVD. An electrocardiogram (ECG) is an inexpensive and noninvasive test that can assist in recognition of arrhythmias and acute coronary syndromes and can be used as a screening tool for CVD. ${ }^{4}$ Other modalities include the echocardiogram, cardiac magnetic resonance imaging (CMRI), and computed tomography $(\mathrm{CT})$. Echocardiography uses reflected ultrasound waves to give information regarding cardiac hemodynamics, function, and structure. It is a powerful tool that is generally used in addition to ECG and chest X-ray for initial CVD assessment. ${ }^{5}$ CMRI uses magnetic resonance imaging to provide clear delineation of the cardiac anatomy, provides a detailed myocardial tissue analysis that aids in diagnosis of specific cardiomyopathies, provides myocardial viability data, and can assist in evaluation of cardiac function. ${ }^{6} \mathrm{CT}$ uses $\mathrm{X}$-rays in the acquisition of data with high spatial resolution. Cardiac CT today can be used to assess atherosclerosis in coronary artery disease, compute a calcium score, evaluate the cardiopulmonary vasculature, and evaluate valves. ${ }^{7}$ However, despite advances in these imaging modalities, each technique requires a technician trained to perform these tests and an experienced operator who can interpret the findings accurately. In addition, these machines are usually only available in large institutions, leaving many underserved areas without such advanced technology to aid in diagnosis and treatment of CVD. ${ }^{1}$ Thus, it is imperative for a physician to develop clinical acuity as well as employ easily available tools such as a stethoscope for a timely diagnosis and treatment of CVD.

\section{The conventional stethoscope}

The stethoscope is an acoustic device that transmits the sounds from the chest piece through an air-filled hollow tube to the listener's ears. The French physician René Laennec first invented it in the 1800s. The design was modest and consisted of a hollow wooden tube that was monaural. Around the same time period, a British physician, Golding Bird, described his version of the stethoscope that comprised flexible tubing that was also monaural. Later, an Irish physician, Arthur Leared, advanced the design and a binaural stethoscope emerged. ${ }^{8}$ The binaural stethoscope has evolved tremendously since then; however, the concept remains the same. The current acoustic binaural stethoscope consists of a hollow tube attached to a chest piece consisting of a wider-based diaphragm and a smaller hollow bell. The diaphragm will transmit higher frequency sounds, whereas the bell will transmit lower-frequency sounds. Thus, the acoustic stethoscope will attenuate sound transmission proportional to the frequency created by the heart sounds. Due to the variation in sensitivity of a human ear, some sounds may not be heard due to low frequency, such as below $50 \mathrm{~Hz}$. This limitation of the acoustic stethoscope has led to the emergence of an electronic device that is far more sophisticated than the original conventional stethoscope (Figure 1).



Figure I Main components of the conventional stethoscope. 


\section{Cardiac and pulmonary auscultation}

It is vital for a physician to appreciate cardiac and pulmonary sounds in his/her patients in order to accurately formulate a diagnosis. Auscultation with a stethoscope provides clinical information that can assist in diagnosing, and altering and directing patient care. Heart sounds are created due to opening and closure of the valves, blood flow through any orifice, flow of blood into the ventricular chambers, and rubbing of the cardiac surfaces. ${ }^{9}$ Heart sound frequencies can be impacted by valve apparatus; however, they normally range from 10 to $200 \mathrm{~Hz} \cdot{ }^{10,11}$ The main areas of auscultation are depicted in Figure 2.

Normal heart sounds comprise the first heart sound (S1), which is produced by the closure of the atrioventricular (AV) valves that are the mitral and tricuspid valves. This corresponds to the end of diastole and the beginning of ventricular systole and precedes the upstroke in the carotid arteries..$^{9-12}$ Variability in auscultation of S1 is described in Figure 3.

The second heart sound (S2) is due to the closure of the aortic valve (A2) and the pulmonic valve (P2) at the conclusion

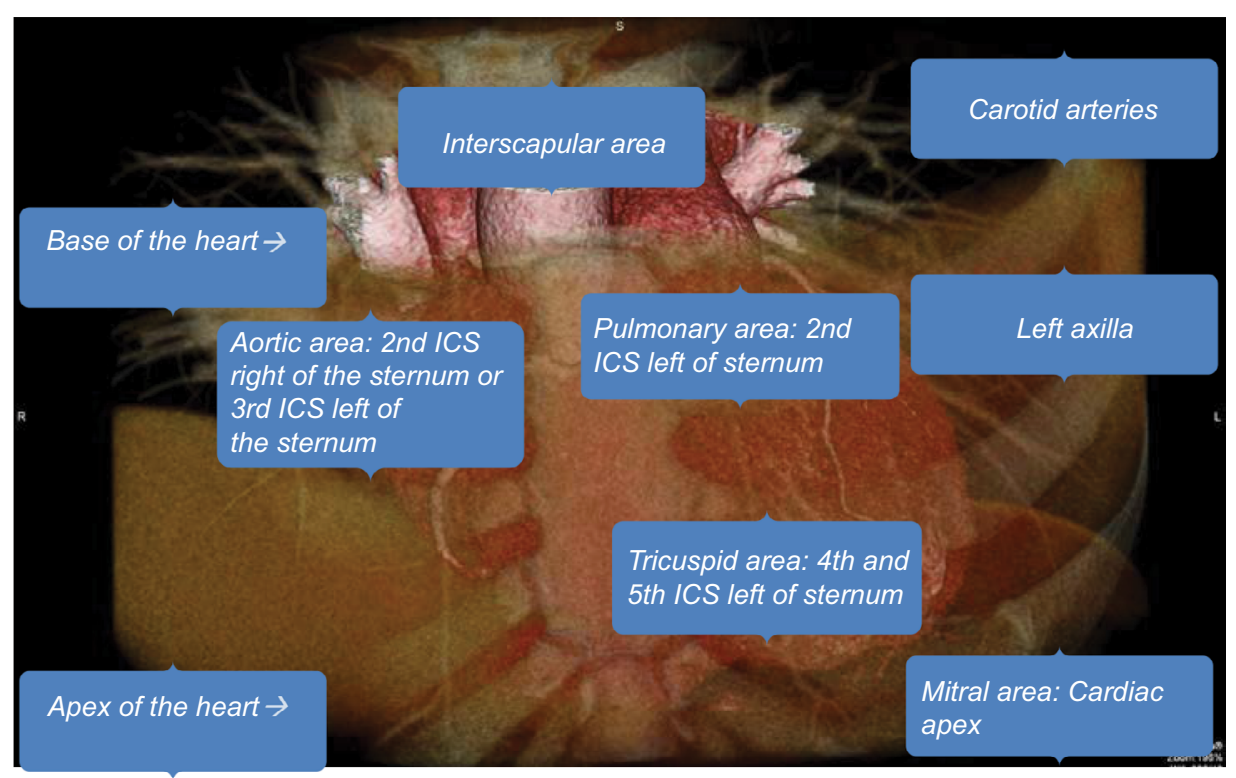

Figure 2 Main areas of cardiac auscultation.

Abbreviation: ICS, intercostal space.

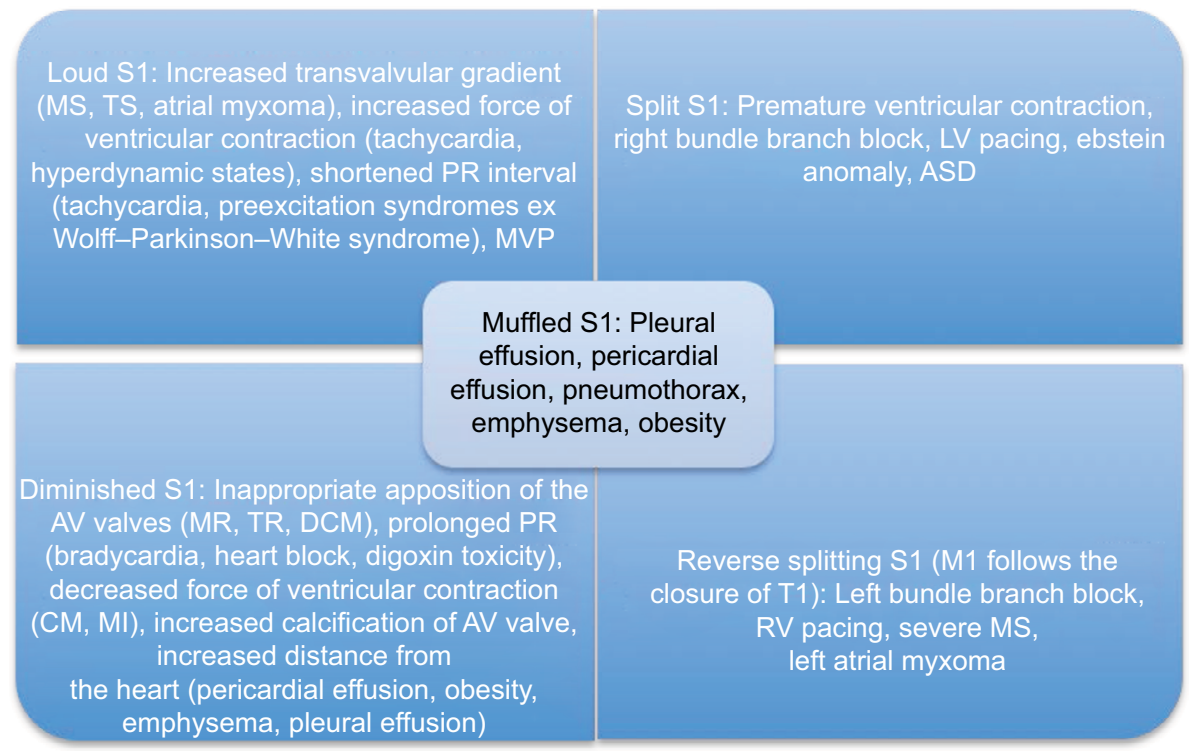

Figure 3 Variability of auscultation of SI. Adapted from Leng S, Tan RS, Chai KT, Wang C, Ghista D, Zhong L. The electronic stethoscope. Biomed Eng Online. 20I5; I4:66. Creative Commons license and disclaimer available from: http://creativecommons.org/publicdomain/zero/I.0/. ${ }^{15}$

Note: $\mathrm{SI}$ is the first heart sound.

Abbreviations: MS, mitral stenosis; TS, tricuspid stenosis; MVP, mitral valve prolapse; LV, left ventricular; ASD, atrial septal defect; AV, atrioventricular; MR, mitral regurgitation; TR, tricuspid regurgitation; DCM, dilated cardiomyopathy; CM, cardiomyopathy; MI, myocardial infarction; MI, mitral valve; TI, tricuspid valve; RV, right ventricular. 
of systole, and it marks the beginning of the diastolic period. Variability in auscultation of S2 is further described in Figure 4.

The third heart sound (S3) occurs in early diastole as the blood enters the ventricle from the atria. It can be normal in individuals up to the age of 40 years; however, if audible in patients of older age, then S3 is pathologic. Pathologically, this can be due to systolic or diastolic ventricular dysfunction, ischemic heart disease, hyperkinetic states (fever, anemia, pregnancy, thyrotoxicosis, AV fistula), VHD, or volume overload. ${ }^{9}$

The fourth heart sound (S4) is a late diastolic sound and is usually produced when the blood from the atria is suddenly decelerated due to a noncompliant left ventricle. Thus, ventricular hypertrophy, ischemic heart disease, ventricular aneurysm, and hyperkinetic states causing forceful atrial contraction can all produce an S4. ${ }^{9-12}$

In addition to the above heart sounds, an opening snap, valvular ejection clicks, and a variety of murmurs can also be appreciated. ${ }^{9}$ A summary of common murmurs is described in Table 1.

Furthermore, another vital component of auscultation is classification of pulmonary pathology to assist in making correct diagnosis. However, accurate interpretation of pulmonary auscultation is subjective and largely dependent on the training of the physician. Auscultation should take place in a quiet room

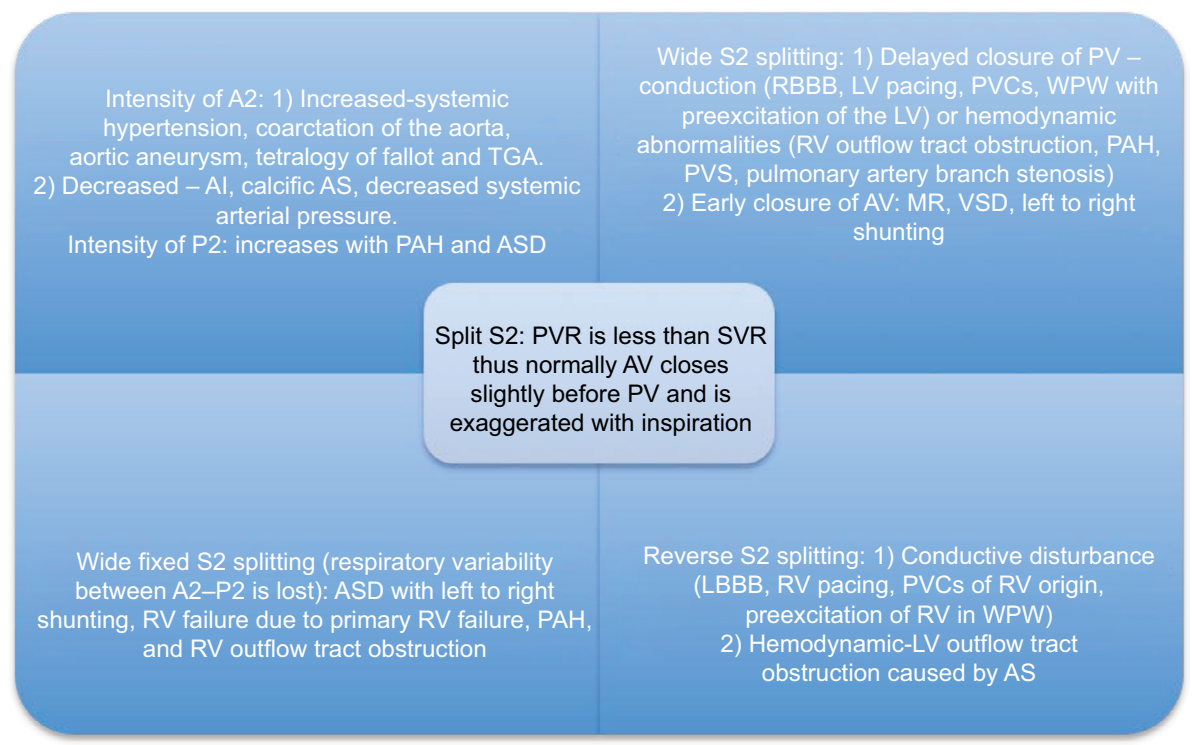

Figure 4 Variability of auscultation of S2. Adapted from Leng S, Tan RS, Chai KT, Wang C, Ghista D, Zhong L. The electronic stethoscope. Biomed Eng Online. 2015 ; I4:66. Creative Commons license and disclaimer available from: http://creativecommons.org/publicdomain/zero/I.0/. ${ }^{15}$

Notes: A2 represents the closure of the aortic valve. P2 is the closure of the pulmonic valve. S2 is the second heart sound.

Abbreviations: TGA, transposition of great arteries; Al, aortic insufficiency; AS, aortic stenosis; PAH, pulmonary arterial hypertension; ASD, atrial septal defect; PV, pulmonic valve; RBBB, right bundle branch block; LV, left ventricular; PVC, premature ventricular contraction; WPW, Wolff-Parkinson-White syndrome; RV, right ventricle; PVS, pulmonic valve stenosis; AV, atrioventricular; MR, mitral regurgitation; VSD, ventricular septal defect; LBBB, left bundle branch block; PVR, pulmonary vascular resistance; SVR, systemic vascular resistance.

Table I Description of common murmurs

\begin{tabular}{|c|c|c|c|c|c|}
\hline Murmur & Location & Quality & Description & Radiation & Pitch \\
\hline$\overline{\text { AS }}$ & Apex/RUS border & Harsh & Systolic, crescendo-decrescendo & Carotid arteries & High \\
\hline AR & RUS border/left 3rd-4th ICS & Blowing & Diastolic, decrescendo & Apex & \\
\hline MS & Apex & Rumbling & $\begin{array}{l}\text { Mid-late diastolic with presystolic } \\
\text { accentuation }\end{array}$ & & Low \\
\hline MR & Apex & Blowing & Holo-systolic & Axilla/base of heart & High \\
\hline PS & Left 2nd ICS & Blowing & Systolic, crescendo-decrescendo & & High \\
\hline TR & Left 4th ICS & Blowing & Diastolic & Left sternal border/xiphoid & High \\
\hline HOCM & Left lower sternal border & Harsh & Mid-late systolic & & High \\
\hline VSD & Left lower sternal border & Harsh & Holo-systolic & & High \\
\hline PDA & Left upper sternal border & Harsh & $\begin{array}{l}\text { Continuous crescendo-decrescendo with } \\
\text { peak about S2 }\end{array}$ & & High \\
\hline MVP & Apex & Blowing & Mid-late systolic & Axilla/base & High \\
\hline
\end{tabular}

Note: Adapted from Leng S, Tan RS, Chai KT, Wang C, Ghista D, Zhong L. The electronic stethoscope. Biomed Eng Online. 20I5; 14:66. Creative Commons license and disclaimer available from: http://creativecommons.org/publicdomain/zero/I.0/.15

Abbreviations: AS, aortic stenosis; RUS, right upper sternal;AR, aortic regurgitation; ICS, intercostal space; MS, mitral stenosis; MR, mitral regurgitation; PS, pulmonic stenosis; TR, tricuspid regurgitation; HOCM, hypertrophic obstructive cardiomyopathy; VSD, ventricular septal defect; PDA, patent ductus arteriosus; MVP, mitral valve prolapse. 
with the patient in a seated position. Auscultation should be done in a symmetrical fashion starting at the apices anteriorly moving to the base and then progression to the posterior chest. Patients should be asked to take deep breaths with their mouth open, while breath sounds should be evaluated for their quality, intensity, and for the presence of unanticipated sounds.

Normal frequency of pulmonary sounds varies from 100 to $1000 \mathrm{~Hz} .{ }^{13}$ Moreover, frequency of wheezing ranges from 100 to $5000 \mathrm{~Hz}$, rhonchus is $150 \mathrm{~Hz}$, coarse crackle is 350 $\mathrm{Hz}$, and fine crackle is $650 \mathrm{~Hz} \cdot{ }^{13}$ Tracheal sounds that are normal are clearly heard in both phases of respiratory cycle, whereas normal lung sound is only heard on inspiration and early phase of expiration. ${ }^{14}$ Wheezing can be heard on inspiration, expiration, or both, and is musical in nature with a high pitch. ${ }^{14}$ Rhoncus may be heard on inspiration, expiration, or both, and is low pitch in nature similar to snoring. ${ }^{14}$ Fine crackles are heard on mid-to-late inspiration and can be heard on expiration, and are usually not transferred to the mouth and unaffected by cough. ${ }^{14}$ Coarse crackle is heard on early inspiration and throughout expiration, is affected by cough, and is transferred to the mouth. ${ }^{14}$ Pleural friction rub and stridor are explosive and high-pitch sounds, respectively, which can also be appreciated during auscultation. ${ }^{14}$ Since multiple pulmonary sounds can be appreciated, and at times simultaneously, digital stethoscope has facilitated in allowing a clinician to accurately understand the pathology behind the sound. As seen by the information that can be gleaned as noted above, the stethoscope serves as an important tool to diagnose a plethora of cardiovascular and pulmonary disease processes by auscultating heart and lung sounds.

\section{The emergence of the digital stethoscope}

A digital stethoscope is able to convert an acoustic sound to electronic signals, which can be further amplified for optimal listening. These electronic signals can be further processed and digitalized to transmit to a personal computer or a laptop. ${ }^{15}$ The diagnostic power provided by digital stethoscope auscultation to a physician can assist in altering management in patient care (Figure 5).

The digital stethoscope consists of three different modules, data acquisition, preprocessing, and signal processing, before the listener can appreciate the auscultated sound. ${ }^{15}$ The data acquisition module involves a microphone and a piezoelectric sensor. It is responsible for filtering, buffering, and amplification of the auscultated sounds, as well as conversion of the acoustic sound to a digital signal. The preprocessing module filters the digital signal and removes any artifacts. These digital data are then forwarded to the signal-processing module, which will package the information in a higher-order classification and cluster the data for a clinical diagnostic decision. ${ }^{15}$

Unlike the acoustic stethoscope, the transducers on a digital stethoscope are of wide variety. One of the transducing

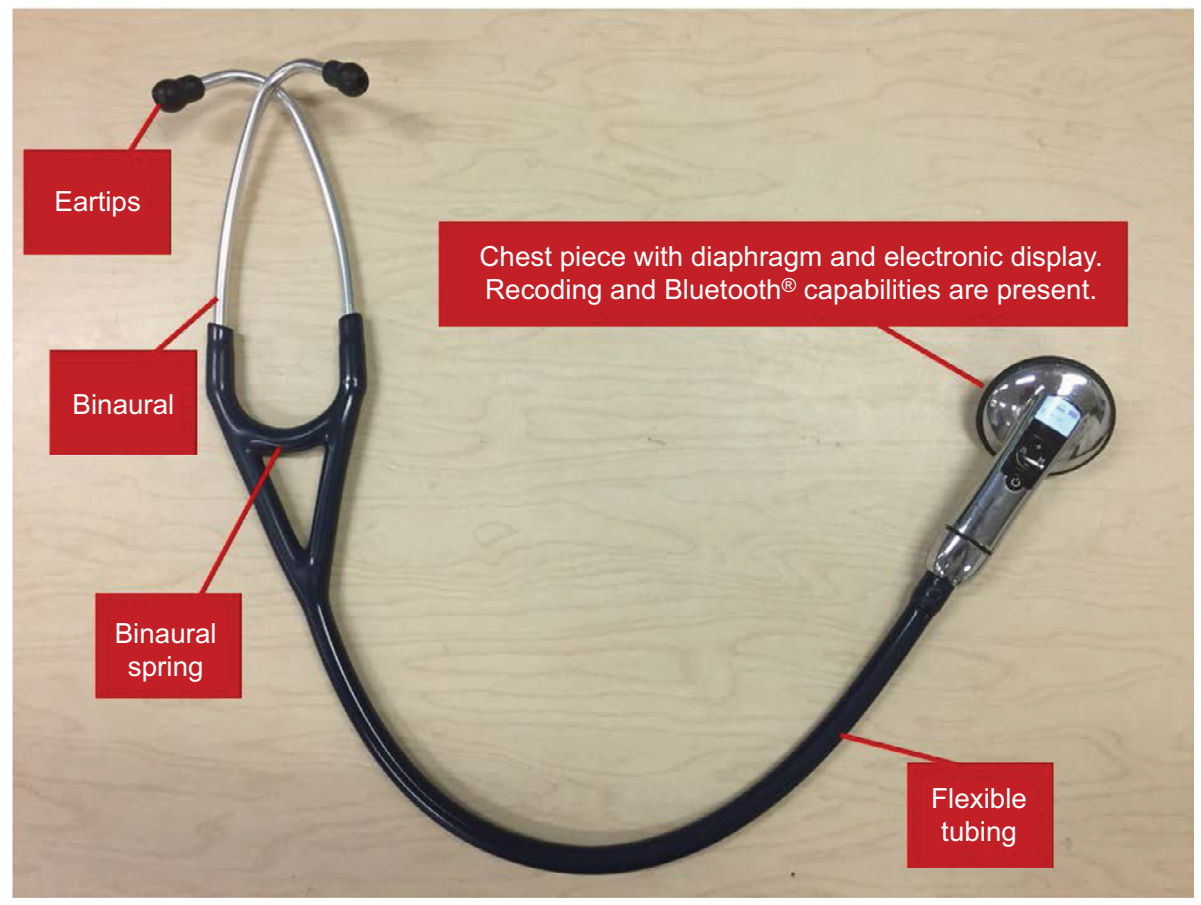

Figure 5 Main components of the digital electronic stethoscope. 
methods involves the microphone in the chest piece. ${ }^{16}$ The sound signals are detected by the stethoscope diaphragm, which is transferred to another diaphragm inside the microphone. This allows for the conversion of a simple and direct acoustic sound to an electrical signal. The signal can then be displayed as a phonocardiogram on an electronic device (Figure 6). However, two diaphragms separated by an air path can result in excessive ambient noise signals and inaccurate electrical signal transfer. ${ }^{15}$

A piezoelectric sensor functions differently to the double-diaphragm mechanism. In a piezoelectric transducer, the sounds picked up by the stethoscope diaphragm cause distortion of a crystal substance that is responsible for then producing an electrical signal. ${ }^{16,17}$ The distortion of the crystal can produce the electrical signal that may not truly capture the original sound ascertained by the diaphragm. ${ }^{15}$

A capacitive micro-electromechanical system is another modality used for a transducer. The diaphragm of the stethoscope is suspended in a nominal capacitance field, where the capacitance changes according to the acoustic pressure formed by the heart sound. The change in capacitance is able to generate an electrical signal. ${ }^{18}$

Currently, almost all available digital stethoscopes allow for selection of different frequency response modes allowing the listener to better hear sounds from the heart, lung, and other areas of the body. There are also multiple mechanisms by which a digital stethoscope can suppress ambient and friction noise to allow the listener to hear sounds that are as original as possible. For instance, the $3 \mathrm{M}^{\circledR}$ Littmann Range offers piezoelectric sensor which uses the ambient noise reduction as an adaptive noise canceller by which it allows to amplify sounds up to 24 times. ${ }^{19}$ The Thinklabs ${ }^{\circledR}$ One Digital stethoscope is able to amplify sounds up to 100 times by applying capacitive transducer. ${ }^{20}$ This technology is able to employ ambient noise reduction but can also use specific heart sound extraction creating a rigorous tool to hear specific heart sounds such as valvular clicks as well as use specific computer algorithms to

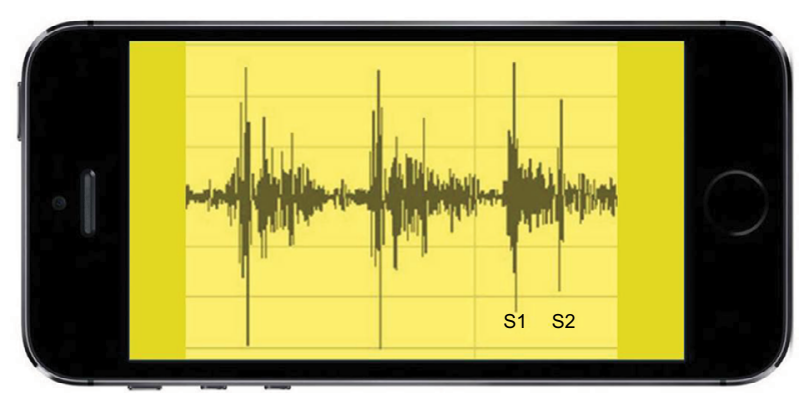

Figure 6 Simulated phonocardiogram of normal SI and S2 heart sounds seen on a portable device through Bluetooth ${ }^{\circledR}$ integration.

Note: SI and S2 represent the first and the second heart sound respectively. extrapolate frequencies to understand pulmonary pathology. ${ }^{20}$ The Welch-Allyn ${ }^{\circledR}$ Elite Electronic Stethoscope allows for a bell mode ranging from 20 to $420 \mathrm{~Hz}$, specifically for heart sounds, and diaphragm mode ranging from 350 to $1900 \mathrm{~Hz}$, which is better used for lung auscultation. ${ }^{21}$ Furthermore, the Ekoscope $^{\circledR}$ stethoscope offers built-in ECG capability, and the ViScope ${ }^{\circledR}$ stethoscope can empower a physician with real-time display of multiple waveforms. ${ }^{22,23} \mathrm{~A}$ digital stethoscope also allows the operator to record the heart sounds and upload them to a computer for further visualization, analysis, and transmission. Additionally, some digital stethoscopes can also be connected to Bluetooth ${ }^{\circledR}$ to wirelessly transmit sound signals to a remote processing unit.

\section{Medical advancements and applications in cardiac and pulmonary auscultation}

Aside from providing increased precision and audible advantage over the conventional stethoscope, digital stethoscopes have also been studied for their utility of screening for obstructive coronary artery disease. Turbulent blood flow occurs due to hemodynamically significant coronary artery disease and manifests as intracoronary murmurs. However, conventional stethoscopes lack the auscultation power to detect these murmurs. We studied the correlation between diagnosis of coronary artery disease using an electronic stethoscope and lesions noted on cardiac CT. ${ }^{24}$ In our prospective single-site study, we wanted to study the accuracy in correlation between the severity and location of coronary artery disease seen on cardiac CT and the microbruits processed by the Cardiac Sonospectrographic Analyzer (CSA; SonoMedica, Vienna, VA, USA) of the electronic stethoscope. A total of 161 patients were studied, and the overall sensitivity of the CSA to ascertain coronary artery disease of $>50 \%$ in any major epicardial artery was $89.5 \%(p<0.001){ }^{24}$

Similarly, Azimpour et al studied similar correlation between audible intracoronary murmurs using a digital stethoscope and the findings on coronary angiography. ${ }^{25} \mathrm{In}$ 123 patients, they were able to ascertain the sensitivity and specificity of acoustic detection of lesions with $>50 \%$ stenosis in any coronary artery to be 0.70 and 0.80 , respectively. ${ }^{25}$

The promise of this new tool to supplement noninvasive imaging in the diagnosis of obstructive coronary disease rests on the premise that small audible signals generated by the turbulent flow in the coronary artery can be appreciated. The CSA, CADence ${ }^{\mathrm{TM}}$, and CADScor ${ }^{\mathrm{B}}$ System are a few examples of acoustic detection systems that are currently being studied to validate against $\mathrm{CT}$ and invasive coronary angiography. ${ }^{26}$ A summary of recent studies is provided in Table 2. 
Table 2 Summary of recent studies utilizing acoustic detection system in diagnosing obstructive coronary artery disease

\begin{tabular}{ll}
\hline Acoustic detection system & Technology \\
\hline CSA SonoMedica model 3.0 & CSA is a noninvasive digital \\
& electronic stethoscope devised to \\
identify microbruits in the frequency \\
range of $400-2700 \mathrm{~Hz}$
\end{tabular}

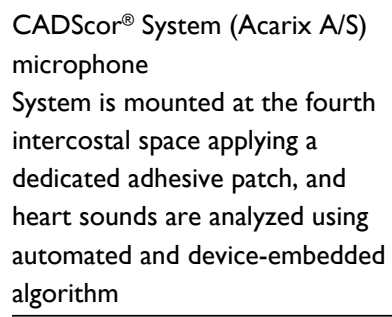

\section{CADence $^{\mathrm{TM}}$ Ironman \\ Designed by Aum Cardiovascular}

Abbreviations: CSA, Cardiac Sonospectrographic Analyzer; CAD, coronary artery

analysis, and final report is sent to

the physician

CAD-score algorithm system sounds combined with clinical risk factors

\author{
Title and results \\ Utility of an advanced digital electronic stethoscope \\ in the diagnosis of coronary artery disease compared \\ with coronary computed tomographic angiography \\ Results: SonoMedica stethoscope with CSA predicted \\ presence of CAD with sensitivity of $89.5 \%$ and \\ specificity of $57.7 \%$ \\ Unpublished study of $1000+$ patients from UCLA \\ 30 \\ CAD can be detected with sensitivity of $81 \%$ and \\ specificity of $83 \%$ \\ Diagnostic performance of an acoustic-based system \\ for coronary artery disease risk stratification \\ Results: Low risk is indicated by a CAD-score value \\ $\leq 20$. At this cutoff, hemodynamically significant CAD \\ was detected with sensitivity of $81 \%$ and specificity of \\ $53 \%$ with NPV of $96 \%$
}

which combines bedside auscultation Results: In a moderate-risk population, obstructive

with echocardiographic data which

is transmitted to a central server for includes acoustic properties of heart
Furthermore, due to the innovation of the digital stethoscope and the capability to transmit the heart sounds wirelessly, there is potential to develop and advance the field of telemedicine. ${ }^{27}$ This will allow physicians to access patients' heart sounds live and alter care and trajectory of their health directly.

Digital aspect of pulmonary auscultation involves recording of the pulmonary sound, computer analysis of the signals obtained, and classification of the sounds based on the frequency analyses. ${ }^{28}$ Computer-based pulmonary sound analysis allows for optimizing and quantifying auscultated lung sounds based on the lung sound as well as the spectral characteristics. The Fourier transform has been the most common analysis tool that has been used to understand pulmonary auscultation. ${ }^{28}$ The Fourier transform is able to degenerate the signal to the frequencies that comprise the signal. Neural network, which is a machine-learning algorithm, can further process this information and classify the different frequencies into pulmonary sounds. ${ }^{28}$ Gurung et al performed a meta-analysis of studies that have tried to understand the prognostic power of combining digital pulmonary auscultation with computerbased algorithms. Although the sample size was limited, they were able to uncover that the specificity and sensitivity of identifying abnormal pulmonary sounds using computerbased algorithms were $85 \%$ and $80 \%$, respectively. ${ }^{28}$

It is also important to note that multiple cardiopulmonary pathologies are connected, and researchers have started to use digital technology to garner accurate diagnosis. For instance, Kaddoura et al used automated machine learning and language-recognition-inspired-speech algorithm to ascertain if the digitally acquired heart sounds were linked to pulmonary hypertension $(\mathrm{PH}){ }^{29}$ The algorithm used was able to closely examine the heart sound and collect information such as amplitude, intensity, shape, and frequency. The heart sounds from $\mathrm{PH}$ patients were compared to non-PH patients, and they discovered the algorithm accurately diagnosed $\mathrm{PH}$ $74 \%$ of the time. ${ }^{29}$ These recent discoveries have opened the door for further research to further optimize the current technology, to ultimately empower the physician to better assist the patient.

\section{Conclusion}

Multiple cardiopulmonary pathologies can be appreciated by the simple mechanism of auscultation. The prevalence of CVD is rising in the world, and multiple CVDs may first manifest as pulmonary symptoms; thus, it is imperative that a physician has the ability to accurately examine a patient even with limited resources. The stethoscope is a powerful tool that is easy to use and allows for direct impact on patient care. With multiple sophisticated advancements made in medicine, which aid in clinical diagnosis and management, none of the modalities compare to the simplicity and vitality of a stethoscope. The emergence of a digital stethoscope has only made this historic tool even more refined. The physician can now hear heart and lung sounds with more accuracy and precision. Through this advancement, there is now potential 
to auscultate for obstructive coronary artery disease, and other bruits and obstructive vascular diseases such as carotid artery stenosis, and examine multiple frequencies that may comprise pulmonary auscultation. Furthermore, there is also potential to drastically impact patient care by appreciating disease processes earlier and to prevent fatal outcome. Medical care can also be provided in areas that are underserved or which do not have medical facilities by applying digital stethoscope technology to telemedicine to allow remote assessment. Although research still needs to be implemented to validate the digital stethoscope further, currently this tool allows the user to be a better diagnostician and thereby deliver better medical care.

\section{Disclosure}

The authors report no conflicts of interest in this work.

\section{References}

1. World Health Organization. Cardiovascular diseases (CVDs). Available from: http://www.who.int/mediacentre/factsheets/fs317/en/. Accessed 31 July 2017.

2. Felner JM. An overview of the cardiovascular system. In: Walker HK, Hall WD, Hurst JW, editors. Clinical Methods: The History, Physical, and Laboratory Examinations. 3rd ed. Boston: Butterworths; 1990.

3. Tavel ME. Cardiac auscultation. A glorious past - but does it have a future? Circulation. 1996;93(6):1250-1253.

4. National Heart, Lung, and Blood Institute. How is heart disease diagnosed? Available from: http://www.nhlbi.nih.gov/health/health-topics/ topics/hdw/diagnosis.html. Accessed 31 July 2017.

5. Esmaeilzadeh M, Parsaee M, Maleki M. The role of echocardiography in coronary artery disease and acute myocardial infarction. J Tehran Heart Cent. 2013;8(1):1-13.

6. De Cobelli F, Pieroni M, Esposito A, et al. Delayed gadoliniumenhanced cardiac magnetic resonance in patients with chronic myocarditis presenting with heart failure or recurrent arrhythmias. $J$ Am Coll Cardiol. 2006;47(8):1649-1654.

7. Nikolaou K, Alkadhi H, Bamberg F, Leschka S, Wintersperger BJ. MRI and $\mathrm{CT}$ in the diagnosis of coronary artery disease: indications and applications. Insights Imaging. 2011;2(1):9-24.

8. Roguin A. Rene Theophile Hyacinthe Laënnec (1781-1826): the man behind the stethoscope. Clin Med Res. 2006;4(3):230-235.

9. The Heart.org/Medscape. Heart sounds. Available from: http://emedicine. medscape.com/article/1894036-overview\#a30. Accessed 31 July 2017.

10. Yoganathan AP, Gupta R, Udwadia FE, et al. Use of the fast Fourier transform for frequency analysis of the first heart sound in normal man. Med Biol Eng. 1976;14(1):69-73.

11. Stein PD, Sabbah HN, Lakier JB, Magilligan DJ Jr, Goldstein D. Frequency of the first heart sound in the assessment of stiffening of mitral bioprosthetic valves. Circulation. 1981;63(1):200-203.
12. Shea MJ. Cardiac Auscultation. Merck Manual Professional Version. Kenilworth: Merck; 2016.

13. Ohshimo S, Sadamori T, Tanigawa K. Innovation in analysis of respiratory sounds. Ann Intern Med. 2016;164(9):638-639.

14. Bohadana A, Izbicki G, Kraman SS. Fundamentals of lung auscultation. N Engl J Med. 2014;370(8):744-751.

15. Leng S, Tan RS, Chai KT, Wang C, Ghista D, Zhong L. The electronic stethoscope. Biomed Eng Online. 2015;14:66.

16. Smith $\mathrm{C}$, inventor; Smith $\mathrm{C}$, assignee. Transducer for sensing body sounds. United States patent US 6661897B2. 2003 Dec 9.

17. Grundlehner B, Buxi D. Methods to characterize sensors for capturing body sounds. Paper presented at: International Conference on Body Sensor Networks (BSN); May 23-25, 2011; Dallas.

18. Kevin TCC, Han D, Ravinder PS, et al. 118-db dynamic range, continuous-time, opened-loop capacitance to voltage converter readout for capacitive MEMS accelerometer. In: IEEE Asian Solid-State Circuits Conference; November 8-10, 2010; Beijing.

19. 3M Littmann Range. Available from: https://www.littmann.com/3M/ en_US/littmann-stethoscopes/. Accessed September 5, 2017.

20. Thinklabs One Digital. Available from: http://www.thinklabs.com/. Accessed September 5, 2017.

21. Welch-Allyn Elite Electronic Stethoscope. Available from: https://www. welchallyn.com/content/welchallyn/americas/en/products/categories/ discontinued-products/physical-exam/stethoscopes.html. Accessed September 5, 2017.

22. Ekoscope. Available from: http://www.ekoscope.com/. Accessed September 5, 2017.

23. ViScope. Available from: http://hdmedicalgroup.com/our-products/ viscope/. Accessed September 5, 2017.

24. Makaryus AN, Makaryus JN, Figgatt A, et al. Utility of an advanced digital electronic stethoscope in the diagnosis of coronary artery disease compared with coronary computed tomographic angiography. Am J Cardiol. 2013;111(6):786-792.

25. Azimpour F, Caldwell E, Tawfik P, Duval S, Wilson RF. Audible coronary artery stenosis. Am J Med. 2016;129(5):515-521.

26. Thomas JL, Winther S, Wilson RF, Bøttcher M. A novel approach to diagnosing coronary artery disease: acoustic detection of coronary turbulence. Int J Cardiovasc Imaging. 2017;33(1):129-136.

27. Lakhe A, Sodhi I, Warrier J, Sinha V. Development of digital stethoscope for telemedicine. J Med Eng Technol. 2016;40(1):20-24.

28. Gurung A, Scrafford CG, Tielsch JM, Levine OS, Checkley W. Computerized lung sound analysis as diagnostic aid for the detection of abnormal lung sounds: a systematic review and meta-analysis. Respir Med. 2011;105(9):1396-1403.

29. Kaddoura T, Vadlamudi K, Kumar S, et al. Acoustic diagnosis of pulmonary hypertension: automated speech-recognition-inspired classification algorithm outperforms physicians. Sci Rep. 2016;6:33182.

30. MEDPAGE TODAY ${ }^{\circledR}$. iMedicalApps: non-invasive devices for heart disease screening. Available from: https://www.medpagetoday.com/ blogs/iltifathusain/67366. Accessed November 14, 2017.

31. Winther S, Nissen L, Schmidt SE, et al. Diagnostic performance of an acoustic-based system for coronary artery disease risk stratification. Heart. Epub 2017 Nov 9.
Medical Devices: Evidence and Research

\section{Publish your work in this journal}

Medical Devices: Evidence and Research is an international, peerreviewed, open access journal that focuses on the evidence, technology, research, and expert opinion supporting the use and application of medical devices in the diagnosis, monitoring, treatment and management of clinical conditions and physiological processes. The identification of novel

\section{Dovepress}

devices and optimal use of existing devices which will lead to improved clinical outcomes and more effective patient management and safety is a key feature. The manuscript management system is completely online and includes a quick and fair peer-review system. Visit http://www. dovepress.com/testimonials.php to read real quotes from authors. 